\title{
Chloroplast microsatellites reveal genetic diver- sity and population structure in natural popula- tions of Himalayan Cedar (Cedrus deodara (Roxb.) G. Don) in India
}

\author{
H. S. Ginwal*, Rajesh Sharma ${ }^{1}$, Priti Chauhan, Kirti Chamling Rai and Santan Barthwal
}

Division of Genetics and Tree Improvement, Forest Research Institute, P.O.I.P.E. Kaulagarh Road, Dehradun-248195, Uttarakhand, India

${ }^{1}$ Himalayan Forest Research Institute, Conifer campus, Panthaghati Shimla (H.P.)

* Corresponding author: H.S.Ginwal, E-mail: ginwalhs@icfre.org; ginwalhs@gmail.com

\begin{abstract}
Himalayan cedar (Cedrus deodara) is one of the most important temperate timber species of Western Himalayas and is considered to be among the endangered conifer species in the region. Knowledge of genetic diversity and population structure will help guide gene conservation strategies for this species. Ten polymorphic chloroplast microsatellites (cpSSR) were used to study genetic diversity and population structure in twenty one natural populations of $C$. deodara throughout its entire distribution range in Western Himalayas. When alleles at each of the 10 loci were jointly analysed, 254 different haplotypes were identified among 1050 individuals. The cpSSRs indicate that $C$. deodara forests maintain a moderately high level of genetic diversity (mean $h=0.79$ ). AMOVA analysis showed that most of the diversity in C. deodara occurs within populations. Bayesian analysis for population structure (BAPS) revealed spatial structuration of the variation ( $22 \%$ of the total variation) and substructuring captured nineteen genetic clusters in the entire divisions of the populations. Most of the populations were clustered independently with minor admixtures. The distribution of genetic diversity and sub-structuring of $C$. deodara may be due to restricted gene flow due to geographic isolation, genetic drift, and natural selection. These findings indicated existence of genetically distinct and different high diversity and low diversity clusters, which are potential groups of populations that require attention for their conservation and management. The results are interpreted in context of future conservation plans for $\mathrm{C}$. deodara.
\end{abstract}

Keywords: : Cedrus deodara, chloroplast microsatellites, genetic diversity, haplotype

\section{Introduction}

The genus Cedrus is believed to have been widely but discontinuously distributed in the Old World during pre quaternary times, according to Pons (1998). Presently, the natural geographic distribution of this genus is limited to Morocco and Algeria for Cedrus atlantica (Atlas cedar); to Lebanon, Syria, and Turkey for Cedrus libani (Lebanon cedar); to Cyprus for Cedrus brevifolia (Cyprus cedar); and to the Himalayan Mountains for Cedrus deodara (Himalayan cedar; Debazac, 1964; Vidakovic 1991) (Dagher-Kharrat et al., 2006). Of these four species, Cedrus deodara commonly referred to as deodar, is one of the most important and endangered temperate timber species of Western Himalayas. The species is found growing right from Afghanistan to Garhwal up to the Valley of Dhauli River below Niti pass at an elevation ranging from $1200 \mathrm{~m}$ to 3050 mamsl (Tewari, 1994). The altitudinal range of deodar is usually higher on the southern than on the northern slopes and the trees grows best and reaches its largest dimensions under cool site conditions on northern aspect. It covers an area of about 0.2 million hectares in India mainly in the states of Jammu and Kashmir, Himachal Pradesh and Uttarakhand.

C. deodara is a large conifer tree of conical shape with spreading horizontal branches. The species is in great demand as building material because of its durability, rot-resistant 
characters, fragrance and fine close grain which is capable of taking a high polish. Due to its multifarious uses it had been subjected to exploitation for centuries. During the British colonial period in India, Deodar wood was extensively used for the construction of barracks, public buildings, bridges, canals and railway cars. Presently natural deodar forests are under great pressure not only due to human impact but also due to the changing climatic conditions. Steady increase in human population, over-exploitation of natural resources, extensive clearing of forests for developmental activity, widespread logging/ lopping and grazing have been responsible for the loss of deodar forests in the Himalayan region. Conservation of this valuable species is immediately required for the sustenance of fragile Himalayan ecosystems.

Long term survival of a species depends on the sustenance of adequate genetic variability within and among populations to accommodate new selection pressure brought about by environmental changes. Genetic diversity is fundamental component of biodiversity and is as critical to sustainability of our natural resources as are species diversity and ecosystem.

Within family Pinaceae, opposite uni-parental inheritance of mitochondrial and chloroplast genomes provides opportunities to assess discontinuity within an effectively haploid genome. The alleles of loci in an organelle genome can be collectively considered as a haplotype because they are not recombining (Terrab et al., 2006). Paternal inheritance of chloroplast genomes in most conifers (Neale and Sederoff, 1989) makes chloroplast microsatellites particularly effective markers for studying mating systems, uni-parental lineages, and gene flow via pollen. Predominant paternal inheritance of chloroplast DNA has been demonstrated in European Abies with previously characterized Pinus thunbergii primers (Vendramin et al., 1996) at two highly variable microsatellite loci (Vendramin and Ziegenhagen, 1997; Ziegenhagen et al., 1998; Vendramin et al., 1999). Intraspecific diversity and population structure in heterologous amplification with these primers have been demonstrated in many other pine species as well (Cato and Richardson, 1996; Morgante et al., 1998).

Knowledge of the variation and distribution of genetic diversity can guide efficient gene conservation sampling designs (Richards et al., 2007). Genetic information is lacking for many species that provide important ecosystem services yet have not had immediate economic value (Schoettle et al., 2012). In spite of the biological and economic importance of $C$. deodara and the existence of severe threat perception on the species, no information is known about its genetic diversity distribution and population structure. In this paper, we have used chloroplast microsatellites to study 1) the levels of haplotypic diversity existing in populations and (2) the population genetic structure of the existing populations of $C$. deodara along the whole distributional area in Himalayas (India). Ten cPSSR loci from 1050 trees from 21 sites in the core distribution range of the species across Himalayan Mountains covering three states viz. Jammu and Kashmir, Himachal Pradesh and Uttarakhand were analyzed. The information will be useful to guide gene conservation strategies for the species

\section{Materials and Methods}

\section{Plant Material}

A total of twenty-one large contiguous deodar populations from three Himalayan states of India were selected for the study that included nine forests from Uttarakhand, ten from Himachal Pradesh and two from Jammu and Kashmir(Table 2, Fig. 1). In order to capture as much as genetic variation as possible within the population, 50 trees were sampled from each population. Selection of the trees was done randomly. The trees considered for collections were well spaced and separated from each other with at least $100 \mathrm{~m}$ apart. The geographical location viz. latitude, longitude and altitude of the sampled trees/populations were recorded. Needles from each of the 50 randomly selected standing adult trees per population were collected and were used for DNA isolation and genotyping.

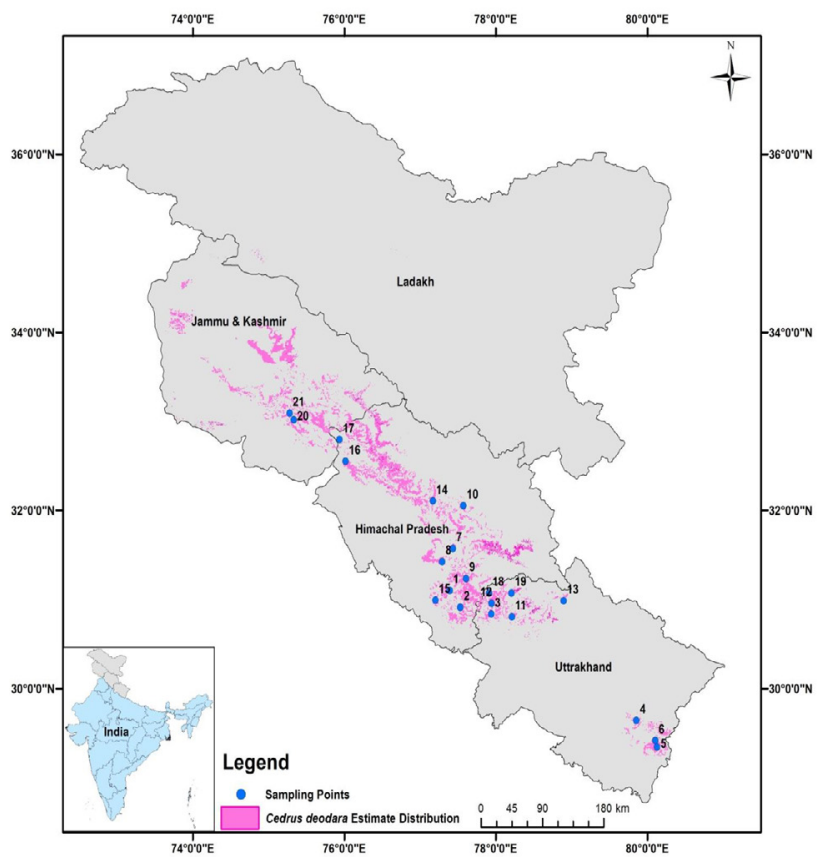

Fig. 1

Map showing location of $21 \mathrm{C}$. deodara populations sampled for genetic diversity analysis in three states in Himalayan mountain range

\section{DNA Extraction and PCR amplification}

Genomic DNA was extracted following protocols by Doyle and Doyle (1990) with some modifications by the addition of $5 \mathrm{mM}$ Ascorbic acid, 2 \% PVP and $0.3 \%$ 2-mercaptoethanol. The washed and dried needles were ground in liquid nitrogen and incubated at $60^{\circ} \mathrm{C}$ for 40 minutes in the modified extraction buffer. Chloroform-isoamyl alcohol (24:1) was added and the g-DNA was precipitated by addition of chilled isopropanol. The g-DNA pellet obtained was washed with $76 \%$ alcohol and 10 $\mathrm{mM}$ ammonium acetate. The pellet was rewashed in $70 \%$ ethanol centrifuged at 13,000 rpm for 15 minutes, vacuum dried 
and re-suspended in 100 $\mu$ l of autoclaved 10mM Tris-EDTA buffer $(\mathrm{pH}$ 8.0). The extracted DNA was quantified and diluted to $15 \mathrm{ng} / \mu \mathrm{l}$ for further use in amplification reactions.

A total of 80 microsatellite primers from $P$. thunbergii, $P$. sylvestris, P. merkussi, P. densiflora, P. resinosa and $P$. taeda were tested by Ginwal et al. (2011) on Cedrus deodara for cross-genus amplification. The primers showing positive amplification were then screened for polymorphism. Of the 80 primers tested on C. deodara, ten chloroplast microsatellite primer pairs showing consistent polymorphism were selected for studying the population genetic structure of twenty one populations of $C$. deodara (Table 1). PCR amplifications were performed by the method of Ginwal et al. (2011), and the PCR-amplified fragments were electrophoresed along with GeneScan ROX 400 internal size standard on an ABI prism 3100 Genetic Analyser (Applied Biosystems). Allele sizes were assigned against the internal size standard and individuals were genotyped using GeneScan analysis software and Genotyper software version 3.7 (Applied Biosystems).

\section{Statistical analyses}

Here we have used the terms "locus" to refer to a cPSSR site, and "allele" to refer to a size-variant at a given cPSSR site. As chloroplast has haploid genomes that are paternally inherited (in most gymnosperms) (Neale and Sederoff, 1989;Watano et al., 1996), hence for the diversity measure, the chloroplast genome was regarded as a single nonrecombining locus and repeat length differences between individuals were scored as haplotypic variants over all cpSSR sites(Parducci et al.,2001). Allele size information at the ten cPSSR loci analysed was used to construct haplotypes. The population genetic parameters viz. effective number of haplotypes ( $n)$, and Nei's unbiased haplotypic diversity $(h)$ were computed as ne $=1 /\left(\sum p_{i}^{2}\right)$, where $p$ is the frequency of the $i$-th haplotype in a population, and by estimating the unbiased genetic diversity (h), which accounts for small population sizes was computed as $h=[n /(n-1)] *$ $\left(1-\sum p_{i}^{2}\right)$, where $\mathrm{n}$ is the number of individuals analyzed in a population and $p_{\mathrm{i}}$ is the frequency of $\mathrm{i}$-th haplotype in a population (Nei, 1987). Unique number of haplotypes $\left(P_{H}\right)$ was calculated using Haplotype Analysis software version 1.05 (Eliades and Eliades, 2009). Gene flow (Nm) which is a measure of effective number of migrants per population per generation, was estimated indirectly from the $G_{S T}$ values at each locus and from the average values over all the loci by applying McDermott and McDonald's (1993) formula Nm =0.5(1-Gst)/Gst for the studied populations using POPGENE 1.32 (Yeh et al., 1999).

An unbiased estimate of Wright's fixation index $F_{\text {ST }}$ (an indicator of the degree of differentiation among populations) was calculated as a measure of genetic differentiation in FSTAT 2.9.3 (Goudet, 2002). The standard error of $F_{\mathrm{ST}}$ was calculated using unbiased jackknife analysis. Analysis of molecular variance was carried out with ARLEQUIN 3.0 software (Excoffier et al., 2005). A hierarchical analysis of variance was used to partition the total variance into covariance components consisting of percentage variation among populations and within populations.
The Bayesian model based approach implemented in BAPS software version 6 (Corander et al., 2013) was used to estimate the hidden population substructure by clustering sample populations into panmictic groups (Corander et al., 2003).We carried out 10 repetitions of the algorithm for each $K$ ranging between 5 and 21 .

\section{Results}

The selected ten cPSSR primers consistently amplified all the twenty one populations of $C$. deodara under standard conditions and were polymorphic in all the populations investigated. The detail of these 10 primers has been presented in Table 1.

Table 1

The detail of chloroplast microsatellite primers that were used for the study.

\begin{tabular}{|c|c|c|c|c|c|c|c|}
\hline \multirow[t]{2}{*}{ Primer } & \multirow[t]{2}{*}{ Sequence (5'-3') } & \multirow{2}{*}{$\begin{array}{l}\text { No. of repeat } \\
\text { units }\end{array}$} & \multirow[t]{2}{*}{ bp } & \multirow[t]{2}{*}{$\mathrm{Tm}$} & \multirow{2}{*}{$\begin{array}{c}\text { Product } \\
\text { size }\end{array}$} & \multicolumn{2}{|c|}{$\begin{array}{c}\text { Genetic } \\
\text { characteristics } \\
\text { of primers }\end{array}$} \\
\hline & & & & & & $\mathbf{n}_{\mathrm{a}}$ & $\mathbf{n}_{\mathrm{e}}$ \\
\hline \multirow{2}{*}{ Pt15169 } & CTTGGATGGAATAGCAGCC & $(\mathrm{C})_{8}(\mathrm{~T})_{8} \mathrm{~A}(\mathrm{~T})_{8}$ & 19 & 58.3 & 118 & 5.0 & 3.08 \\
\hline & GGAAGGGCATTAAGGTCATTA & $(\mathrm{C})_{s}(\mathrm{~T})_{14}$ & 21 & & & & \\
\hline \multirow{2}{*}{ Pt26081 } & CCCGTATCCAGATATACTTCCA & $(\mathrm{T})_{14}$ & 22 & 58 & 112 & 5.0 & 3.16 \\
\hline & TGGTTTGATTCATTCGTTCAT & $(\mathrm{T})_{11}$ & 21 & & & & \\
\hline \multirow{2}{*}{ Pt30204 } & TCATAGCGGAAGATCCTCTTT & $(\mathrm{A})_{12}(\mathrm{G})_{10}$ & 21 & 58 & 145 & 4.0 & 3.13 \\
\hline & CGGATTGATCCTAACCATACC & $(\mathrm{A})_{9}(\mathrm{G})_{8}$ & 21 & & & & \\
\hline \multirow{2}{*}{ Pt36480 } & TTTTGGCTTACAAAATAAAAGAGG & $(\mathrm{T})_{11}$ & 24 & 58.1 & 147 & 5.0 & 3.07 \\
\hline & AAATTCCTAAAGAAGGAAGAGCA & $(\mathrm{T})_{9}$ & 23 & 58.1 & 148 & 5.0 & 3.36 \\
\hline Pt71936 & AAAACCGTACATGAGATTCCC & $(\mathrm{T})_{14}$ & 21 & & & & \\
\hline \multirow{2}{*}{ РСР9434 } & AAACTGACGTAGATGCCATGG & $(\mathrm{A})_{10}$ & 21 & 58.5 & 131 & 5.0 & 2.59 \\
\hline & GCGGTATGAGGGAAGAAGC & & 19 & & & & \\
\hline \multirow[t]{2}{*}{ РСР26106 } & AATCCGACAAAAAAGATTCGG & $(\mathrm{A})_{14}$ & 21 & 58.5 & 149 & 5.0 & 3.56 \\
\hline & GCTCCATTTCACGTGGTTG & & 19 & 585 & 115 & 40 & 302 \\
\hline РСР36567 & $\begin{array}{l}\text { AAAAGAGGAGGAAAAACACCTT } \\
\text { AAGAGCAGACAAGTAAGGGGC }\end{array}$ & (1) & 21 & & & & \\
\hline \multirow{2}{*}{ PCP45071 } & ACTGGTCTGATCGACCCAAT & $(\mathrm{T})_{15}$ & 20 & 58.5 & 149 & 5.0 & 3.47 \\
\hline & TTCTACACTTGCGGAAACCC & & 20 & & & & \\
\hline \multirow{3}{*}{ PCP100842 } & TCAATACAAATGATGGGAGTCG & $(\mathrm{A})_{12}$ & 22 & 58.5 & 146 & 4.0 & 2.85 \\
\hline & TTTTGCCATATCCTGAAACTCC & & 22 & & & & \\
\hline & Mean & & & & & 4.7 & 3.13 \\
\hline
\end{tabular}

The most variable cpSSR loci across 21 studies populations were Pt15169, Pt26081, Pt36480, Pt71936, PCP9434, PCP26106 and PCP45071 with 5 alleles each (Table 1). The number of alleles per polymorphic locus ranged from 4-5 for all the 10 primer pairs with a mean of 4.7. The effective number of alleles ranged from 2.59 to 3.56 with a mean of 3.13. Gene flow Nm calculated was 1.951, indicating moderate levels of gene flow.

\section{Haplotype distribution}

When alleles at each of the 10 loci were jointly analyzed, 254 different haplotypes were identified among 1050 individuals. Table 2 shows the genetic characteristic of the chloroplast haplotypes based on the $10 \mathrm{cpSSR}$ loci in $21 \mathrm{C}$. deodara populations. The lowest value of haplotypic diversity $(h=0.71)$ was observed in population 17 from Churah Forest Division (HP) and the highest value of haplotypic diversity $(h=0.89)$ was 
observed in population 21 from Batote (JK). Estimates of the effective number of haplotypes and haplotypic diversity averaged across all the populations were 4.99 and 0.79 respectively. In intra population analysis, an average of twelve private haplotypes was detected. The population 1 from Theog Forest Division (H.P.) contained the highest number (23) of private haplotypes, while population 10 from Chopal Forest Division (H.P.) had the lowest (2) (Table 2). Inter population analysis revealed high levels of total genetic diversity $\left(\mathrm{H}_{\mathrm{T}}\right)$ and consequently, high levels within each populations $\left(H_{s}\right)$. However, diversity due to genetic differentiation $\left(D_{S T}\right)$ was comparatively low in all the populations (Figure 2).

Table 2

Haplotypic distribution of twenty one populations of $C$. deodara

\begin{tabular}{|c|c|c|c|c|c|c|c|}
\hline \multirow[b]{2}{*}{ Code } & \multicolumn{2}{|c|}{ Population detail } & \multirow{2}{*}{$\begin{array}{l}\text { Geographical } \\
\text { coordinates } \\
\text { Latitude }\left({ }^{\circ} \mathrm{N}\right) \\
\text { Longitude } \\
\left({ }^{\circ} \mathrm{E}\right) \\
\end{array}$} & \multirow[b]{2}{*}{$\begin{array}{l}\text { Alt (m) } \\
\text { amsl }\end{array}$} & \multirow{2}{*}{ 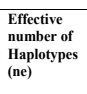 } & \multirow{2}{*}{$\begin{array}{l}\text { Haplotypic } \\
\text { diversity (h) }\end{array}$} & \multirow{2}{*}{$\begin{array}{c}\begin{array}{c}\text { Number of } \\
\text { private } \\
\text { haplotypes } \\
\left(\mathrm{P}_{\mathrm{H}}\right)\end{array} \\
\end{array}$} \\
\hline & Forest area & Location & & & & & \\
\hline 1 & $\begin{array}{l}\text { Theog Forest } \\
\text { Division (HP) }\end{array}$ & $\begin{array}{l}\text { Vintage point } \\
\text { bifurcation of road } \\
\text { rainfall }\end{array}$ & $\begin{array}{l}31^{\circ} 03^{\prime} 45^{\prime \prime} \mathrm{N} \\
77^{\circ} 19^{\prime} 62^{\prime \prime} \mathrm{E}\end{array}$ & $\begin{array}{l}2212- \\
2478\end{array}$ & 6.596 & 0.849 & 23 \\
\hline 2 & $\begin{array}{l}\text { Chopal Forest } \\
\text { Division (HP) }\end{array}$ & Sairan & $\begin{array}{l}30^{\circ} 53^{\prime} 99^{\circ \mathrm{N}} \\
77^{\circ} 31^{\prime} 56^{\circ} \mathrm{E}\end{array}$ & $\begin{array}{l}2162- \\
2415\end{array}$ & 6.545 & 0.848 & 8 \\
\hline 3 & $\begin{array}{l}\text { Chakrata } \\
\text { Forest } \\
\text { Division (UK) }\end{array}$ & $\begin{array}{l}\text { Kathyyan Beat and } \\
\text { Molta range }\end{array}$ & $\begin{array}{l}30^{\circ} 53^{\circ} 077^{\prime \prime N} \mathrm{~N} \\
77^{\circ} 56^{4} 47^{\circ} \mathrm{E}\end{array}$ & $\begin{array}{l}2074- \\
2294\end{array}$ & 4.513 & 0.779 & 14 \\
\hline 4 & $\begin{array}{l}\text { Jageswar } \\
\text { Forest } \\
\text { Division (UK) }\end{array}$ & $\begin{array}{l}\text { Purvipanwanula, } \\
\text { Jageswar } 2 \mathrm{~A}, 2 \mathrm{~B}\end{array}$ & $\begin{array}{l}29^{\circ} 38229^{\circ} \mathrm{N} \\
79^{\circ} 51^{\prime} 25^{\prime \prime} \mathrm{E}\end{array}$ & $\begin{array}{l}\begin{array}{l}1783- \\
1992\end{array} \\
{ }^{2}\end{array}$ & 4.417 & 0.774 & 6 \\
\hline 5 & $\begin{array}{l}\text { Champawat } \\
\text { Forest } \\
\text { Division (UK) }\end{array}$ & $\begin{array}{l}\text { Malaharaswar, } \\
\text { Ghatudedarwani }\end{array}$ & $\begin{array}{l}29^{\circ} 20^{\prime} 28^{\prime \prime N} \mathrm{~N} \\
80^{\circ} 07^{\prime} 46^{\prime \prime} \mathrm{E}\end{array}$ & $\begin{array}{l}1555- \\
1667\end{array}$ & 4.153 & 0.760 & 5 \\
\hline 6 & $\begin{array}{l}\text { Lohaghat } \\
\text { Forest } \\
\text { Division (UK) }\end{array}$ & $\begin{array}{l}\text { Lohaghat reserve } \\
\text { and chidiadhunga }\end{array}$ & $\begin{array}{l}29^{\circ} 2128^{\circ} \mathrm{N} \\
80^{\circ} 06^{\prime} 46^{\prime \prime} \mathrm{E}\end{array}$ & $\begin{array}{l}1533- \\
1680\end{array}$ & 4.132 & 0.759 & 8 \\
\hline 7 & $\begin{array}{l}\text { Division ( (KK) } \\
\text { Anni Forest } \\
\text { Division (HP) }\end{array}$ & Luhri and Chowai & $\begin{array}{l}31^{\circ} 27^{\prime} 48^{\prime \prime N} \mathrm{~N} \\
77^{\circ} 27^{\prime} 20^{\prime \prime E}\end{array}$ & $\begin{array}{l}1938- \\
2120\end{array}$ & 5.198 & 0.808 & 12 \\
\hline 8 & $\begin{array}{l}\text { Karsog Forest } \\
\text { Division (HP) }\end{array}$ & Seri & $\begin{array}{l}31^{\circ} 20^{\prime} 31^{\prime \prime N} \\
77^{\circ} 18^{\prime} 29^{\prime \prime} \mathrm{E}\end{array}$ & $\begin{array}{l}1909- \\
2391\end{array}$ & 4.808 & 0.793 & 20 \\
\hline 9 & $\begin{array}{l}\text { Rampur Forest } \\
\text { Division (HP) }\end{array}$ & Bhurja, Punan & $\begin{array}{l}31^{\circ} 1^{\prime} 52^{\prime \prime N} \\
77^{\circ} 35^{\prime} 09^{\prime \prime} \mathrm{E}\end{array}$ & $\begin{array}{l}2267- \\
2520\end{array}$ & 4.266 & 0.766 & 22 \\
\hline 10 & $\begin{array}{l}\text { Chopal Forest } \\
\text { Division (HP) }\end{array}$ & Chopal & $\begin{array}{l}31^{\circ} 56^{\prime} 96^{\prime \prime N} \\
77^{\circ} 34^{\circ} 92^{\prime \prime} \mathrm{E}\end{array}$ & $\begin{array}{l}2313- \\
2503\end{array}$ & 5.040 & 0.802 & 2 \\
\hline 11 & $\begin{array}{l}\text { Barkot Forest } \\
\text { Division (UK) }\end{array}$ & Beyali & $\begin{array}{l}33^{\circ} 05^{\prime} 27^{\prime \prime N} \\
75^{\circ} 19^{\prime} 47^{\prime \prime E}\end{array}$ & $\begin{array}{l}1847- \\
2017\end{array}$ & 3.597 & 0.723 & 14 \\
\hline 12 & $\begin{array}{l}\text { Uttarkashi } \\
\text { Forest } \\
\text { Division (UK) }\end{array}$ & Harshil & $\begin{array}{l}30^{\circ} 53^{\prime} 07^{\prime \prime N} \mathrm{~N} \\
77^{\circ} 56^{\prime} 47^{\prime \prime E}\end{array}$ & $\begin{array}{l}2414- \\
2685\end{array}$ & 3.698 & 0.730 & 18 \\
\hline 13 & $\begin{array}{l}\text { Division (UK) } \\
\text { Uttarkashi } \\
\text { Forest } \\
\text { Division (UK) }\end{array}$ & Gangotri & $\begin{array}{l}30^{\circ} 59^{\prime} 07^{\prime \prime N} \mathrm{~N} \\
78^{\circ} 53^{\prime} 54^{\prime \prime} \mathrm{E}\end{array}$ & $\begin{array}{l}2887- \\
3087\end{array}$ & 4.902 & 0.797 & 6 \\
\hline 14 & $\begin{array}{l}\text { Kullu Forest } \\
\text { Division (HP) }\end{array}$ & $\begin{array}{l}\text { Naggarjhir and } \\
\text { Phetavan }\end{array}$ & $\begin{array}{l}32^{\circ} 06^{\prime} 24^{\prime \prime} \mathrm{N} \\
77^{\circ} 09^{\prime} 74^{\prime \prime} \mathrm{E}\end{array}$ & $\begin{array}{l}1618- \\
1942\end{array}$ & 5.459 & 0.818 & 9 \\
\hline 15 & $\begin{array}{l}\text { Wild life } \\
\text { Shimla Forest } \\
\text { Division (HP) }\end{array}$ & $\begin{array}{l}\text { Binoo (C2 and } \\
\text { C8) }\end{array}$ & $\begin{array}{l}30^{\circ} 58^{\prime} 89^{\prime \prime} \mathrm{N} \\
77^{\circ} 12^{\prime} 17^{\prime \prime} \mathrm{E}\end{array}$ & $\begin{array}{l}1952- \\
2089\end{array}$ & 5.507 & 0.819 & 4 \\
\hline 16 & $\begin{array}{l}\text { Wildlife } \\
\text { Chamba } \\
\text { Forest } \\
\text { Division (HP) }\end{array}$ & Kalatop & $\begin{array}{l}32^{\circ} 33^{\prime} 04^{\prime \prime} \mathrm{N} \\
76^{\circ} 01^{\prime} 08^{\prime \prime} \mathrm{E}\end{array}$ & $\begin{array}{l}2181- \\
2461\end{array}$ & 3.731 & 0.733 & 16 \\
\hline 17 & $\begin{array}{l}\text { Churah Forest } \\
\text { Division (HP) }\end{array}$ & $\begin{array}{l}\text { Khani Reserve } \\
\text { forest }\end{array}$ & $\begin{array}{l}32^{\circ} 47^{\prime} 42^{\prime \prime N} \\
75^{\circ} 55^{\prime} 78^{\prime \prime} \mathrm{E}\end{array}$ & $\begin{array}{l}1785- \\
2090\end{array}$ & 3.492 & 0.714 & 21 \\
\hline 18 & $\begin{array}{l}\text { Tons Forest } \\
\text { Division (UK) }\end{array}$ & Gokul beat & $\begin{array}{l}31^{\circ} 04^{\prime} 19^{\prime \prime N} \mathrm{~N} \\
77^{\circ} 54^{\prime} 47^{\prime \prime} \mathrm{E}\end{array}$ & $\begin{array}{l}2291- \\
2462\end{array}$ & 5.230 & 0.810 & 19 \\
\hline 19 & $\begin{array}{l}\text { Tons Forest } \\
\text { Division (UK) }\end{array}$ & $\begin{array}{l}\text { Sankri (Purti } \\
\text { block and Saumd) }\end{array}$ & $\begin{array}{l}31^{\circ} 04^{\prime \prime} 17^{\prime \prime N} \\
78^{\circ} 12^{\prime} 32^{\prime \prime} \mathrm{E}\end{array}$ & $\begin{array}{l}1963- \\
2092\end{array}$ & 5.353 & 0.814 & 8 \\
\hline 20 & Patnitop (JK) & Udhampur & $\begin{array}{l}33^{\circ} 04^{\prime} 24^{\prime \prime N} \\
75^{\circ} 19^{\prime} 46^{\prime \prime} \mathrm{E}\end{array}$ & $\begin{array}{l}1942- \\
2076\end{array}$ & 5.020 & 0.802 & 13 \\
\hline 21 & Batote (JK) & Batote & $\begin{array}{l}33^{\circ} 05^{\prime} 34^{\prime \prime N} \\
75^{\circ} 19^{\prime} 50^{\circ} \mathrm{E}\end{array}$ & $\begin{array}{l}1989- \\
2017\end{array}$ & 9.259 & 0.893 & 6 \\
\hline & Mean & & & & 4.996 & 0.790 & 12.095 \\
\hline
\end{tabular}

\section{AMOVA analysis}

The twenty one populations of $C$. deodara showed significant levels of genetic differentiation quantified using ARLEQUIN. The hierarchical level of molecular variance among populations was confirmed by analysis of molecular variance (AMOVA). The total molecular variance was portioned into two levels i.e. among populations and within populations (Table 3). AMOVA analysis revealed that $77.29 \%$ of the variation was present among individuals within populations, $22.71 \%$ was due to differences among populations. Variance estimates were based

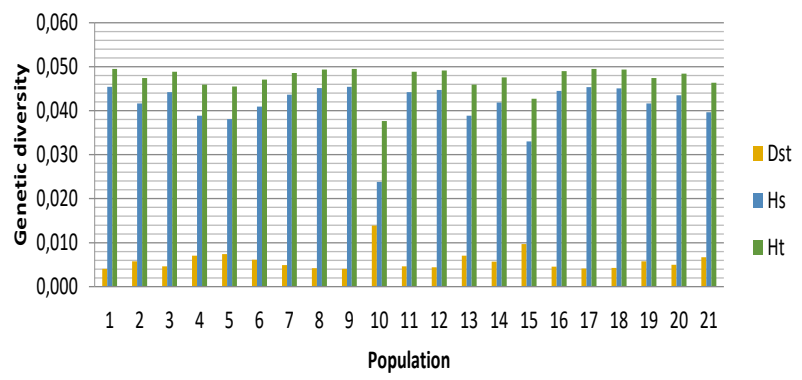

Fig.2

Inter population genetic parameters

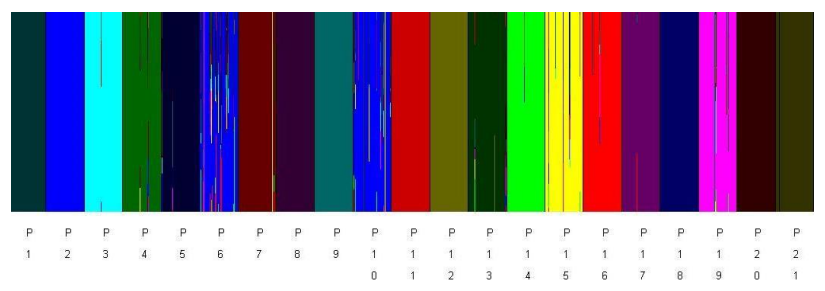

Fig. 3

Inferred clusters of individual genotypes with highest posterior probabilities of likelihood values obtained at $K=19$. The colours represent the most likely ancestry of the cluster from which the genotype or partial genotype was drawn. The populations (P1 to P21) are separated by black vertical lines.

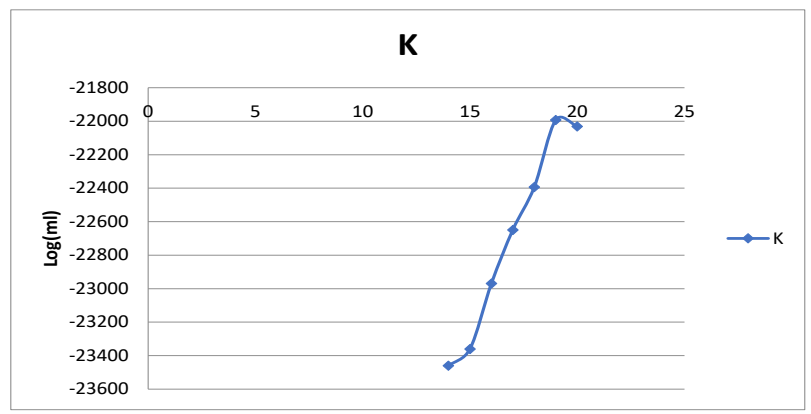

Fig. 4

Log-marginal likelihood distribution of $\mathrm{K}$ genetic clusters using BAPS for all populations of Cedrus deodara.

on 1000 permutations. Accordingly, the differences between individuals variation within the populations was statistically significant $(\mathrm{P}<0.001)$.The populations revealed relatively high genetic differentiation $\left(F_{\mathrm{ST}}=0.22\right)$ and inter-population diversity (Table 3).

\section{Population Structure analysis}

BAPS analysis identified nineteen different clusters as the best partition of the whole sample(all the 10 best marginal log-likelihood values were for $K=19$, with the most optimal value of -21992.1578) (Figure 4). The distribution of the populations into different clusters identified by the BAPS analysis has been presented in Table 5. The 21 populations of $C$. deodara were 
Table 3

Hierarchical analysis of molecular variance (AMOVA) partitioning variance into among population and within populations

\begin{tabular}{lllllll}
\hline $\begin{array}{l}\text { Source of } \\
\text { variation }\end{array}$ & $\mathrm{df}$ & $\mathrm{SS}$ & $\mathrm{MSS}$ & $\begin{array}{l}\text { Variance } \\
\text { components }\end{array}$ & $\begin{array}{l}\text { Percentage } \\
\text { variation }\end{array}$ \\
\hline $\begin{array}{l}\text { Among } \\
\text { population }\end{array}$ & 20 & 877.066 & 101.289 & 0.858 & 22.709 & $F_{\mathrm{SC}}=0.17$ \\
$\begin{array}{l}\text { Within } \\
\text { populations }\end{array}$ & 1029 & 3004.860 & 2.920 & 2.920 & 77.290 & $F_{\mathrm{ST}}=0.22$ \\
$\begin{array}{l}\text { Total } \\
\text { df = Degree of freedom, SS }=\text { sum of squares, MSS = mean sum of squares }\end{array}$
\end{tabular}

Table 4

Distribution of the populations into the nineteen cpSSR clusters identified by the BAPS analysis.

\begin{tabular}{cccccccccccccccccccc}
\hline $\begin{array}{c}\text { Population } \\
\text { Code }\end{array}$ & 1 & 2 & 3 & 4 & 5 & 6 & 7 & 8 & 9 & 10 & 11 & 12 & 13 & 14 & 15 & 16 & 17 & 18 & 19 \\
\hline 1 & - & - & - & - & - & - & - & - & - & - & - & - & - & - & - & - & - & 50 & - \\
2 & - & - & - & - & - & - & 49 & - & - & - & - & - & - & - & 1 & - & - & - & - \\
3 & - & - & - & - & - & - & - & - & - & 50 & - & - & - & - & - & - & - & - & - \\
4 & - & - & - & - & - & - & - & - & - & - & 49 & - & - & - & 1 & - & - & - & - \\
5 & - & - & - & - & - & - & - & - & 1 & - & - & - & - & - & 48 & 1 & - & - & - \\
6 & - & - & 1 & - & - & - & - & - & - & - & - & - & 1 & - & 47 & 1 & - & - & - \\
7 & - & - & - & - & - & - & - & - & - & - & - & 49 & 1 & - & - & - & - & - & - \\
8 & - & - & - & - & - & - & - & - & - & - & - & - & - & - & - & - & 50 & - & - \\
9 & - & - & - & - & - & - & - & - & - & - & - & - & - & 50 & - & - & - & - & - \\
10 & - & - & 4 & - & - & - & - & - & - & - & - & - & - & - & 45 & 1 & - & - & - \\
11 & - & - & - & - & - & - & - & - & - & - & - & - & - & - & - & - & - & - & 50 \\
12 & - & - & - & - & - & 50 & - & - & - & - & - & - & - & - & - & - & - & - & - \\
13 & - & - & 50 & - & - & - & - & - & - & - & - & - & - & - & - & - & - & - & - \\
14 & - & - & - & - & 50 & - & - & - & - & - & - & - & - & - & - & - & - & - & - \\
15 & 45 & - & - & - & 1 & - & - & - & 1 & - & - & - & - & - & 2 & - & 1 & - & - \\
16 & - & - & - & - & - & - & - & - & - & - & - & - & 48 & - & 2 & - & - & - & - \\
17 & - & 50 & - & - & - & - & - & - & - & - & - & - & - & - & - & - & - & - & - \\
18 & - & - & - & 50 & - & - & - & - & - & - & - & - & - & - & - & - & - & - & - \\
19 & - & - & - & - & - & - & - & - & - & - & - & - & - & - & 3 & 47 & - & - & - \\
20 & - & - & - & - & - & - & - & 48 & - & - & - & - & 2 & - & - & - & - & - & - \\
21 & - & - & - & - & - & - & - & - & 48 & - & - & - & - & - & 1 & 1 & - & - & - \\
Over all & 45 & 50 & 55 & 50 & 51 & 50 & 49 & 48 & 49 & 50 & 49 & 49 & 52 & 50 & 150 & 51 & 51 & 50 & 50 \\
\hline
\end{tabular}

divided into 19 clusters for both the individual and admixture models (Figure 3). For $K=19$, the clusters formed were biologically most consistent with populations 5 and 6 falling under the same cluster 15 both belonging to Uttarakhand, similarly clusters 8 and 9 are characterized by the populations of Jammu and Kashmir (J.K.). The results showed that most of the populations were clustered independently with higher levels of admixtures occurring in populations belonging to Uttarakhand.

\section{Discussion}

Our results clearly showed that $C$. deodara maintains a high level of genetic diversity in Western Himalayas. This is in accordance with the general observation in most coniferous species (Vendramin et al., 1998; Clark et al., 2000; Parducci et al., 2001; Ribeiro et al., 2001; Gomez et al., 2003; Hansen et al., 2005). The high genetic diversity in C. deodara in the present study is in accordance with the very high genetic diversity of 0.95 reported earlier in Cedrus atlantica with the help of chloroplast microsatellite markers. The highest level of haplotypic diversity was concentrated in population 21 from Batote, Jammu and Kashmir, which should therefore deserve particular attention in genetic conservation programs for Himalayan cedar. Apart from maintaining higher genetic diversity, the populations of $C$. deodara are also significantly structured. As expected, owing to the greater degree of isolation, population divergence or isolation is higher for $C$. deodara $\left(F_{\mathrm{ST}}=0.22\right)$, which was higher than that reported for $C$. atlantica $\left(F_{\mathrm{ST}}=\right.$ 0.107) (Terrab et al., 2006) and $P$. radiata (Wu et al., 1998), but was lower in comparison to Himalayan Chir pine (P. roxburghii) $\left(F_{\text {ST }}=0.32\right.$, Chauhan 2011).

The main reason for the high genetic diversity observed in C. deodara may be associated with its long life cycle outcrossing mating system, wind pollination behaviour and high regeneration potential (Mehta et al., 2015; Tiwari et al., 2018). Another factor that has an important bearing is its large geographic distribution range, which had major differences in geography, climate and habitat conditions. Himalayan ridge top ecosystems are considered to be more sensitive to climate change as they are characterized by uniform sunlight exposure, but variation in altitude give birth to climatic differences and subsequent vegetation changes (Tiwari et al., 2018). Most of the variation in C. deodara lies within the populations as the species is a temperate cross pollinated with wind and gravity-dispersed seeds, this finding may fit with the general observation that woody, perennial and outbreeding species maintain most of their variation within populations. These results are compatible with those involving other woody perennial, out breeding plant species, especially conifers (Hamrick et al., 1992). In a review, Nybom and Bartish (2000) assembled AMOVA derived $F_{S T}$ values for about 100 plant species and confirmed the tendency of gymnosperms to have lower values of population differentiation than angiosperms. The $F_{\text {ST }}$ value for $C$. deodara in this study is comparatively lower than other conifer species with discontinuous distributions and large populations. For instance, P. cembra has an $F_{\mathrm{ST}}$ value of 0.32 (Szmidt, 1982), Scottish $P$. sylvestris populations recorded $F_{S T}$ value of 0.37 (Sinclair et al., 1997), and P. torreyana has a value of 1.0 with different alleles fixed in each one of its two populations (Ledig and Conkle, 1983). Several studies have shown that differentiation generally increases with distance (Dumolin-Lapègue et al., 1997; Grivet \& Petit, 2002a; Palmé and Vendramin 2002; Heuertzet al., 2004).

The Bayesian analysis methodology takes the number of populations as well as the allele frequencies in each population as random variables thereby detecting the hidden population substructure by clustering sampled populations into a panmictic group (Corander et al., 2004; Elaides et al., 2011). The Bayesian analysis identified 19 independent ancestries and some of them appeared within populations as well. Furthermore, the clusters showed very limited and in some populations no admixture indicating that they were highly differentiated probably due to weak pollen-mediated gene flow between populations. Since the populations collected were geographically far apart it is possible that the populations have not admixed in recent history. Gene flow is the factor that is usually 
supported by effective cross-pollination, seed and pollen dispersal mechanism (Lee et al., 2000) and presence of strong supporters for gene exchange. The levels of gene flow in out crossing, wind pollinated tree species such as $C$. deodara are expected to be high, but the value $(\mathrm{Nm}=1.95)$ is considerably lower than reported for conifer species, such as Larix lyallii (Nm = 3.5; Khasa et al., 2006), P. taeda $(\mathrm{Nm}=1.87-6.71$; Al-Rababah and Williams, 2002) and P. strobus ( $\mathrm{Nm}=3.10$, Mehes et al., 2009). Gene flow and genetic differentiation are a vice-versa phenomenon and are co-dependent, if one component increases the other decreases. It appears there are limited restrictions in the gene flow in the natural range of distribution of this species resulting in heterogeneous genetic structures. Restricted gene flow among populations may limit migration of genes or genotypes to new suitable habitats as the climate continues to change (Schoettle et al., 2012). Furthermore, if climate suitable for $C$. deodara no longer exists, genetic diversity of the species will be deteriorated by the loss of the more genetically diverse high-elevation populations.

The genetic diversity mainly came from individuals in different populations. Results suggest a lack of genetic connectivity across the distribution range regardless of site type. Geographical isolation is the main reason for the differences among the populations.The Himalayan vegetation landscape particularly the north-western and central Himalayan region have highly fragmented forest patches as compared to the eastern Himalayan region (Reddy et al., 2013). The conversion of vegetation cover to non-forest area by human activity further increased forest fragmentation (Sharma and Roy 2007). Expansion in agriculture and the built-up area are the major drivers of forest cover change and fragmentation resulting in forest degradation with implications for biodiversity, habitat ecosystem services, and people's livelihoods (Batar et al., 2017). One of the primary consequences of habitat fragmentation is the loss of connection among fragments with similar characteristics. Many species are therefore disconnected and this may lead to genetic isolation, minimizing the possibility of exchanging alleles, which means a low gene flow (Nm) among populations. This creates local population structure and tends to increase genetic divergence among fragments (Konzen, 2014).

The investigation revealed that the deodar forests of Jammu and Kashmir and majority of the deodar forests of Himachal Pradesh are rich in haplotypic diversity (values $>8$ ) particularly the populations 1,2 , and 21 . On the other hand, the forests originating from Uttarakhand state possess a lower level of diversity, particularly in two populations viz. 11 and17. The total area of deodar forests in India is estimated around 203,263 ha (Himachal Pradesh 69,872 ha, Jammu and Kashmir 113000 ha and Uttarakhand 20,391 ha) (Tewari, 1994). Looking at its range of distribution, it is naturally found in the northwestern Himalayas from Kashmir to Garhwal (part of Uttarakhand) covering the states of Jammu and Kashmir, Himachal Pradesh and north western part of Uttarakhand. In Uttarakhand, only the Tons forest division (populations 18 and 19) of Tehri Garhwal Himalayas forms the eastern natural limit in the sampling range, hence it is obvious that the these regions would have higher haplotypic diversity as is evident. Beside this, C. deodara has become naturalized beyond this limit in Uttarakhand in many patches at Lohaghat (population 6), Champawat (population 5), Jageshwar (population 4) etc. These populations fall in one genetic cluster and encompass highadmixed ancestry of diverse genotypes as compared to other populations. This is possibly due to human mediated movement of genotypes in these populations and seems more like plantation rather than natural population. Due to geographical distances these forests were unable to receive the gene movement from other high diversity forests.

\section{Conservation implications}

Conservation priority should be given to populations with 1) high level of genetic diversity and 2) possessing unique haplotypes. A distinct high diversity group evident here is represented by eight populations $(1,2,7,10,18,19,20,21)$ wherein the haplotypic diversity levels are> 0.80 .Second, some of the populations $(1,8,9,12,17,18)$ possess highest private or rare haplotypes. Such populations are more likely to be in need of genetic rescue and/or restoration. If these populations become extinct, the diversity of $C$. deodara would be diminished /deteriorated. Combining these two categories, there will be eleven populations viz. 1, 2, 7, 8, 9, 10, 12, 17, 18, 19 and 21 that would require special attention for their conservation. Augmented gene flow from these genetically diverse and distinct populations needs to be considered as a way of increasing fitness and the adaptive potential of populations. Among these populations, some appear to have higher priority than others based on both the uniqueness and diversity level. Populations 1 and 18 had the highest contribution to the total gene diversity as well as rare haplotypes. Therefore, these two populations should have the top priority in conservation planning and implementation. Timber harvesting may be prohibited in these populations and sustainable forest management strategies should be implemented in order to maintain the genetic diversity of these populations. An in situ/ex-situ conservation strategy would be effective to protect these habitats. In order to make best possible use of the high genetic diversity populations, it will be important to harvest any available seed from these populations and be used for plantations and infusing diversity in less diversity populations and for their ex situ conservation.

\section{Acknowledgements}

We would like to thank the Directors of Forest Research Institute Dehradun and Himalayan Forest Research Institute Shimla for providing all infrastructure and logistic support for conducting this work. This research was funded by Indian Council of Forestry Research and Education, Dehradun in the form of a research project to Dr. H.S. Ginwal and Dr. Rajesh Sharma. We would like to thank state forest departments of Uttarakhand, Himachal Pradesh and Jammu and Kashmir for providing help in field surveys and sample collection. 


\section{References}

Al-Rabab'Ah MA and Williams CG (2002) Population dynamics of Pinus taeda L. based on nuclear microsatellites. Forest Ecology and Management, 163: 263-271. https://doi.org/10.1016/s0378-1127(01)00584-9

Batar A, Watanabe T and Kumar A (2017) Assessment of Land-Use/Land-Cover Change and Forest Fragmentation in the Garhwal Himalayan Region of India. Environments, 4(2). https://doi.org/10.3390/environments4020034

Cato SA and Richardson TE (1996) Inter- and Intra-specific polymorphism at chloroplast SSR loci and the inheritance of plastids in Pinus radiate D. Don. Theoretical and Applied Genetics, 93(4):587- 592. https://doi.org/10.1007/s001220050319

Chauhan P (2011) Molecular genetic analysis of Chir pine (Pinus roxburghii Sarg.) through microsatellite markers. Ph.D thesis submitted to Forest Research Institute, Deemed University, Dehradun, India.

Clark CM, Wentworth TR and O'Malley DM (2000) Genetic discontinuity revealed by chloroplast microsatellites in eastern North American Abies (Pinaceae). American Journal of Botany, 87: 774-782.https://doi.org/10.2307/2656885

Corander J, Cheng L, Marttinen P, Siren J and Tang J (2013) BAPS: Bayesian analysis of population structure manual v. 6.0. Accessed 9 August 2018 http://www.helsinki.fi/bsg/software/BAPS/

Corander J, Waldmann P and Sillanpaa MJ (2003) Bayesian analysis of genetic differentiation between populations. Genetics, 163, 367-374.

Corander J, Waldmann P, Marttinen P and Sillanpaa MJ (2004) BAPS 2: enhanced possibilities for the analysis of genetic population structure. Bioinformatics, 20: 2363-2369. https://doi.org/10.1093/bioinformatics/bth250

Dagher-Kharrat MBD, Mariette S, Lefevre F, Fady B, March GG, Plomion C and Sovoure A (2006) Geographical diversity and genetic relationships among Cedrus species estimated by AFLP. Tree Genetics and Genomics, 3(3): 275 285. https://doi.org/10.1007/s11295-006-0065-x

Debazac EF (1964) Manuel des conifères. ENGREF, Nancy

Doyle JJ and Doyle JL (1990) Isolation of plant DNA from fresh tissue. Focus, 12: 13-15.

Dumolin-Lapégue S, Demesure B., Corre LEV., Fineschi S, Petit RJ (1997) Phylogeographic structure of white oaks throughout the European continent. Genetics, 146: 1475-1487.

Echt C, Vernol DEL., Anzidei M and Vendramin GG (1998) Chloroplast microsatellites reveal population genetic diversity in red pine, Pinus resinosa Ait. Molecular Ecology.7: 307-316. https://doi.org/10.1046/j.1365-294x.1998.00350.x

Eliades N-G, Gailing O, Leinemann L, Fady B and Finkeldey R (2011) High genetic diversity and significant population structure in Cedrus brevifolia Henry, a narrow endemic Mediterranean tree from Cyprus. Plant Systematics and Evolution, 294: 185-198. https://doi.org/10.1007/s00606-011-0453-z

Eliades N-G and Eliades DG (2009) HAPLOTYPE ANALYSIS: Software for analysis of haplotype data. Distributed by the authors.Forest Genetics and Tree Breeding, Georg-August University Goettingen, Germany. URL

Excoffier L, Laval G and Schneider S (2005) ARLEQUIN ver. 3.0: An integrated software package for population genetics data analysis. Evolutionary Bioinformatics Online, 1: 47-50. https://doi.org/10.1177/117693430500100003

Ginwal HS, Chauhan P, Barthwal S, Sharma A and Sharma R (2011) Cross-Species Amplification and Characterization of Pinus Chloroplast Microsatellite Markers in Cedrus deodara Roxb. Silvae Genetica, 60 (2): 65-69. https://doi.org/10.1515/sg-2011-0009

Gomez A, Gonzalez-Martinez SC, Collada C, Climent J and Gil I (2003) Complex population genetic structure in the endemic Canary Island pine revealed using chloroplast microsatellite markers. Theoretical and Applied Genetics, 107: 1123-1131. https://doi.org/10.1007/s00122-003-1320-2

Goudet J (2002) FSTAT: A program to estimate and test gene diversities and fixation indices. Version 2.9.3.2. http://www.unil.ch/izea/softwares/fstat.htm/

Grivet D and Petit RJ (2002) Phylogeography of the common ivy (Hedera sp.) in Europe: genetic differentiation through space and time. Molecular Ecology, 11: 1351-1362. https://doi.org/10.1046/j.1365-294x.2002.01522.x

Hamrick JL, Godt MJW and S. L. Sherman-Broyles SL (1992) Factors influencing levels of genetic diversity in woody plant species. New Forests, 6: 95-124. https://doi.org/10.1007/bf00120641

Hansen OK, Kjaer ED and Vendramin GG (2005) Chloroplast microsatellite variation in Abies nordmanniana and simulation of causes for low differentiation among populations. Tree Genetics and Genomes, 1: 116-123.

https://doi.org/10.1007/s11295-005-0016-y

Heuertz M, FINESCHI S and Anzidei M (2004) Chloroplast DNA variation and postglacial recolonisation of common ash (Fraxinus excelsior L.) in Europe. Molecular Ecology, 13: 3423-3438.

https://doi.org/10.1111/j.1365-294x.2004.02333.x

Khasa DP, Jaramillo-Correa JP, Jaquish B and Bosquet J (2006) Contrasting microsatellite variation between subalpine larch and western larch, two closely related species with different distribution patterns. Molecular Ecology, 15:3907-3918. https://doi.org/10.1111/j.1365-294x.2006.03066.x

Konzen E (2014) Towards conservation strategies for forest tree endangered species: the meaning of population genetic statistics. Advances in Forestry Science, 1 (1): 45-51.

Ledig FT and Conkle MR (1983) Gene diversity and genetic structure in a narrow endemic torrey pine (Pinus torreyana Parry ex. Carr). Evolution, 37: 79-85. https://doi.org/10.1111/j.1558-5646.1983.tb05515.x

Lee SL, Wickneswari R, Mahani MC and Zakri AH (2000) Genetic diversity of a tropical tree species, Shorea leprosula Miq. (Dipterocarpaceae), in Malaysia: Implications for conservation of genetic resources and tree improvement. Biotropica, 32: 213-224. https://doi.org/10.1111/j.1744-7429.2000.tb00464.x Maheshwari P and Biswas C (1970) Cedrus. Botanical Monograph No.5 Series. Council of Scientific and Industrial Research, New Delhi.

McDermott JM and McDonald BA (1993) Gene flow in plant pathosystems. Annual Review Phytopathology, 31: 353- 373. https://doi.org/10.1146/annurev.py.31.090193.002033

Mehes M, Nkongolo KK and Michael P (2009) Assessing genetic diversity and structure of fragmented populations of eastern white pine (Pinus strobus) and western white pine (Pinus monticola) for conservation management. Journal of Plant Ecology, 2(3): 143-151. https://doi.org/10.1093/jpe/rtp016

Mehta JP, Shreshthamani and Bhatt VP (2015) Regeneration potential and distribution pattern of tree species along altitudinal gradient in Central Himalaya. International Journal of Scientific and Research Publications, 5 (5): 1-6.

Morgante M, Felice N and Vendramin GG (1998) Analysis of hypervariable chloroplast microsatellites in Pinus halepensis reveals a dramatic genetic bottleneck. In A. Karp, P. G. Isaac, and D. S. Ingram [eds.], Molecular tools for screening biodiversity, 407-412. Chapman and Hall, London, UK. https://doi.org/10.1007/978-94-009-0019-6 73

Neale DB and SEDEROFF RR (1989) Paternal inheritance of chloroplast DNA and maternal inheritance of mitochondrial DNA in loblolly pine. Theoretical and Applied Genetics, 77: 212-216. https://doi.org/10.1007/bf00266189

Nei M (1987) Molecular Evolutionary Genetics. Columbia University Press, New York.

Nybom H and Bartish IV (2000) Effects of life history traits and sampling strategies on genetic diversity estimates obtained with RAPD markers in plants. Perspectives in Plant Ecology, Evolution and Systematics, 3: 93-114. https://doi.org/10.1078/1433-8319-00006

Palmé A and Vendramin GG (2002) Chloroplast DNA variation, postglacial recolonization and hybridization in hazel, Corylus avellana. Molecular Ecology, 11, 1769-1779. https://doi.org/10.1046/j.1365-294x.2002.01581.x

Parducci L, Szmidt AE, Madaghiele A, Anziei M and Vendramin GG (2001) Genetic variation at chloroplast microsatellites (cpSSRs) in Abies nebrodensis (Lojac.) Mattei and three neighboring Abies species. Theoretical and Applied Genetics, 102: 733-740. https://doi.org/10.1007/s001220051704

Pons A (1998) L'histoire du genre Cedrusd'aprés les donnéespaléobotaniquesdisponibles. For Méditerr, 14: 236-242

Reddy CS, Sreelekshmi S, Jha CS and Dadhwal VK (2013) National assessment of forest fragmentation in India: Landscape indices as measures of the effects of fragmentation and forest cover change. Ecological Engineering, 60, 453e464. http://dx.doi.org/10.1016/j.ecoleng.2013.09.064

Ribeiro MM, Plomion C, Petit R, Vendramin GG and Szmidt AE (2001) Variation in chloroplast single-sequence repeats in Portuguese maritime pine (Pinus pinaster Ait.). Theoretical and Applied Genetics, 102: 97-103. https://doi.org/10.1007/s001220051623

Richards CM, Antolin MF, Reilley A, Poole J and Walters C (2007) Capturing genetic diversity of wild populations for ex situ conservation: Texas wild rice (Zizania texana) as a model. Genetic Resources and Crop Evolution, 54(4): 837-848. https://doi.org/10.1007/s10722-006-9167-4 
Schoettle AW, Goodrich BA, Hipkins V, Richards C and Kray J (2012) Geographic patterns of genetic variation and population structure in Pinus aristata, Rocky Mountain bristlecone pine. Canadian Journal of Forest Research, 42: 23-37. https://doi.org/10.1139/x11-152

Sharma R and Bhondge S (2016) Unpredictable Reproductive Behavior of Cedrus deodara (Roxb.) G. Don. Journal of Forest and Environmental Science, 32: 113-119. https://doi.org/10.7747/jfes.2016.32.2.113

Sharma S and Roy PS (2007) Forest fragmentation in the Himalaya: A Central Himalayan case study. Int. J. Sustain. Dev. World Ecol. 14, 201-210. https://doi.org/10.1080/13504500709469720

Sinclair WT, Moncur JD and Ennos RA (1997) Multiple origins for Scots pine (Pinus sylvestris L.) in Scotland: evidence from mitochondrial DNA variation. Heredity, 80: 233-240. https://doi.org/10.1046/j.1365-2540.1998.00287.x

Szmidt AE (1982) Genetic variation in isolated populations of stone pine (Pinus cembra). SilvaeFennica, 16: 196-200.

Terrab A, Paun O, Talavera S, Tremetsberger K, Arista M, Stuessy TF (2006) Genetic diversity and populations of Moroccan Atlas Cedar (Cedrus atlantica; Pinaceae) determined with cpSSR markers. American Journal of Botany, 93(9): 1274-1280. https://doi.org/10.3732/ajb.93.9.1274

Tewari DN (1994) A monograph on deodar (Cedrus deodara (Roxb.) G. Don). International Book Distributors, Dehra Dun, India, pp. 3, 14 .

Tiwari OP, Rana YS, Krishan RAM, Sharma CM and Bhandari BS (2018) Regeneration dynamics, population structure, and forest composition in some ridge forests of the Western Himalaya, India. Forest Science and Technology, 14: 2, 66-75.https://doi.org/10.1080/21580103.2018.1447517

Troup RS (1921) The Silviculture of Indian Trees. Vol. III. Clarendon Press, Oxford. https://doi.org/10.5962/bhl.title. 24363

Vendramin GG, Anzidei M, Madaghiele M and Bucci G (1998) Distribution of genetic diversity in Pinus pinaster Ait. as revealed by chloroplast microsatellites. Theoretical and Applied Genetics, 97: 456-463. https://doi.org/10.1007/s001220050917

Vendramin GG and Ziegenhagen B (1997) Characterization and inheritance of polymorphic plastid microsatellites in Abies. Genome, 40: 857-864. https://doi.org/10.1139/g97-811

Vendramin GG, Degen B, Petit RJ, Anzidie M, Madaghiele A and Ziegenhagen B (1999) High level of variation at Abies alba chloroplast microsatellite loci in Europe. Molecular Ecology, 8: 1117-1126. https://doi.org/10.1046/j.1365-294x.1999.00666.x

Vendramin GG, Lellilr Rossi P and Morgante M (1996) A set of primers for the amplification of 20 chloroplast microsatellites in Pinaceae. Molecular Ecology, 5: 595-598. https://doi.org/10.1046/j.1365-294x.1996.00111.x

Vidakovic M (1991) Conifers. In: Brekalo B (Ed) Morphology and variation. Graficki Zavod, Hrvatske, pp 129-136.

Watano Y, Imazu M and Shimizu T (1996) Spatial distribution of cpDNA and mtDNA haplotypes in a hybrid zone between Pinus pumila and P. parviflora var. Pentaphylla (Pinaceae). Journal of Plant Research, 109: 403-408. https://doi.org/10.1007/bf02344555

Wright S (1978) Evolution and the Genetics of Population, Variability Within and Among Natural Populations. The University of Chicago Press, Chicago.

Wu JW, Krutovskii KV and Strauss SH (1998) Abundant mitochondrial genome diversity, population differentiation and convergent evolution in pines. Genetics, 150: 1605-1614.

Yeh FC, Yang RC and Boyle TBJ (1999) PopGene Version 1.31: Microsoft windows based Freeware for Population Genetic Analysis. University of Albert, Edmonton. http://www.ualberta.ca/fyeh/

Ziegenhagen B, Scholz F, Madaghiele A and Vendramin GG (1998) Chloroplast microsatellites as markers for paternity analysis in Abies alba. Canadian Journal of Forestry Research, 28: 317-321. https://doi.org/10.1139/x97-213 